\title{
Dynamic decompression of the lateral femoral cutaneous nerve to treat meralgia paresthetica: technique and results
}

\author{
Martijn J. A. Malessy, MD, PhD,,2 Job Eekhof, MD, PhD, ${ }^{2}$ and Willem Pondaag, MD, PhD',2 \\ ${ }^{1}$ Leiden University Medical Center, and ${ }^{2}$ Alrijne Medical Center, Leiden, The Netherlands
}

OBJECTIVE The results of lateral femoral cutaneous nerve (LFCN) decompression to treat idiopathic meralgia paresthetica (iMP) vary widely. Techniques to decompress the LFCN differ, which may affect outcome, but in MP it is unknown to what extent. The authors present a new technique using dynamic decompression and discuss the outcomes.

METHODS A retrospective cohort study was performed in a consecutive series of 19 cases. The goal of decompression was pain relief and recovery of sensation. The plane ventral to the LFCN was decompressed by cutting the fascia lata and the inferior aspect of the inguinal ligament. The plane dorsal to the LFCN was decompressed by cutting the fascia of the sartorius muscle. Subsequently, the thigh was brought in full range of flexion and extension/abduction. The authors identified and additionally cut fibers that tightened and caused compression at various locations of the LFCN during movement in all patients, referring to this technique as dynamic decompression. Postoperatively, an independent neurologist scored pain and sensation on a 4-point scale: completely resolved, improved, not changed, or worsened. Patients scored their remaining pain or sensory deficit as a percentage of the preoperative level. Statistical assessment was done using ANOVA to assess the association between outcome and duration of preoperative symptoms, BMI, and length of follow-up.

RESULTS In 17 of the 19 cases (89\%), the pain and/or paresthesia completely resolved. Patients in the remaining 2 cases (11\%) experienced $70 \%$ and $80 \%$ reduction in pain. Sensation completely recovered in 13 of the 19 cases $(69 \%)$. In 5 of the 19 cases $(26 \%)$ sensation improved, but an area of hypesthesia remained. Four of these 5 patients indicated a sensory improvement of more than $75 \%$, and the remaining patient had $50 \%$ improvement. Sensation remained unchanged in 1 case (5\%) with persisting hypesthesia and mild hyperesthesia. There was no significant impact of preoperative symptom duration, BMI, and length of follow-up on postoperative outcome.

CONCLUSIONS Dynamic decompression of the LFCN is an effective technique for the treatment of iMP. Most patients become completely pain free and sensation recovers considerably.

https://thejns.org/doi/abs/10.3171/2018.9.JNS182004

KEYWORDS lateral femoral cutaneous nerve; meralgia paresthetica; dynamic decompression; peripheral nerve; nerve surgery

$\mathrm{P}$ AIN, paresthesia, and abnormal skin sensation on the lateral side of the thigh result from pathology of different origins in the course of spinal nerves L2 and L3 to the lateral femoral cutaneous nerve (LFCN). The clinical diagnosis of idiopathic meralgia paresthetica (iMP) is made when a herniated lumbar disc or a pelvic tumor is excluded and the symptoms are not caused by trauma or surgical intervention. In the $1960 \mathrm{~s}$, it became generally accepted that the cause of iMP is compression of the LFCN in its location beneath the lateral portion of the inguinal ligament at the site of the anterior superior iliac spine (ASIS). ${ }^{21}$
Treatment for iMP either is conservative or consists of 1) local injection with a combination of steroids with an anesthetic; 2) pulsed radiofrequency (PRF); 3) spinal cord stimulation; or 4) surgery. ${ }^{34}$ The objective evidence base for treatment choices is weak. ${ }^{23}$ Two surgical techniques are currently in use, namely LFCN decompression and neurectomy. It is unknown whether one technique is superior to the other. ${ }^{23,35}$

Over time, different techniques of decompression have been described, consisting of cutting 1) the inguinal ligament over the femoral nerve; ${ }^{32}$ 2) the division of the upper blade of the inguinal ligament and deep fascia of the

ABBREVIATIONS ASIS = anterior superior iliac spine; iMP = idiopathic meralgia paresthetica; LFCN = lateral femoral cutaneous nerve; PRF = pulsed radiofrequency; RCT $=$ randomized controlled trial.

SUBMITTED July 11, 2018. ACCEPTED September 26, 2018.

INCLUDE WHEN CITING Published online December 7, 2018; DOI: 10.3171/2018.9.JNS182004. 
thigh:26 3 ) a small portion of the fibrous origin of the internal abdominal oblique muscle: ${ }^{27}$ and 4) a slot in the crest of the ilium posterior to the ASIS..$^{28}$ Other techniques are medial transposition of the LFCN ${ }^{17}$ and minimally invasive neurolysis. ${ }^{8}$ Textbooks and handbooks usually provide fairly superficial information regarding the technique of decompression. . $^{6,16,20,22,24,25,30,37,42}$

Many articles have reported the results of LFCN decompression. In the latest Cochrane review on treatment for iMP, ${ }^{23}$ only 9 articles were considered of high quality and were included. ${ }^{2,13,15,29,31,33,38,41,44}$ For unclear reasons, 2 studies that actually met the criteria were not used.,39 Unfortunately, $6^{2,13,15,31,33,41}$ of these $11^{2,4,13,15,29,31,33,38,39,41,44}$ papers could not be used to determine the relationship of the decompression technique and its outcome for the following reasons. First, patients with idiopathic as well as symptomatic MP were grouped. ${ }^{2,13}$ It is known that the etiology underlying MP symptoms affects the outcome of decompression. ${ }^{4}$ Second, revision decompression or neurectomy was performed following failed decompression, but was scored as one treatment. ${ }^{33}$ Third, results of decompression or neurectomy were pooled. ${ }^{41}$ Fourth, nerves originating from the femoral nerve were decompressed, ${ }^{41}$ which makes it unlikely that these nerves were the LFCN.$^{43}$ Fifth, patients were children ${ }^{15}$ or concerned only those with symptomatic MP. ${ }^{31}$

The 5 remaining papers comprise well-delineated groups. Pain relief was obtained in $30 \%$ to $82 \%$ of the patients. ${ }^{4,29,38,39,44}$ Unfortunately, only 3 of these 5 articles included details regarding the surgical technique performed and were thus eligible for relating technique to outcome. ${ }^{4,38,39}$ Benezis et al. performed ventral decompression of the LFCN by opening the fascia lata and by sectioning the inguinal ligament. Complete pain relief was obtained in $64 \%$ of the patients. ${ }^{4}$ Siu and Chandran and Son et al. additionally addressed 3 structures dorsal to the LFCN, namely a posterior sling of fascia from the inguinal ligament, the tendinous arc from the iliac fascia, and the distal deep fascia of the thigh. Complete relief was achieved in $73 \%$ and $82 \%$ of the patients, respectively. ${ }^{38,39}$ Recently, the effect on outcome of implementing technical modifications was published. ${ }^{17}$ The number of patients per group was small and follow-up incomplete, making a meaningful interpretation impossible. However, the applied surgical modifications ${ }^{17}$ mirror the trend in time..$^{4,38,39}$ Initially, only ventral decompression was performed, and later dorsal decompression was added. Better outcomes were obtained with more extensive decompression.

The large variation in the reported outcome of LFCN decompression for iMP is striking., $429,38,39,44$ This might be explained not only by inclusion bias, but also by the applied surgical technique. In any case, so far all decompressions have been done supine with the leg neutral. Our technique of nerve decompression differs in this aspect. As a routine, we always check whether compression of the nerve occurs in the dynamics of movement.

More specifically, in decompressing the LFCN to treat iMP, we include moving the thigh. Structures that cause compression in flexion, extension, and abduction are precisely identified and cut as well. We call this technique dynamic decompression. To our knowledge, this technique
TABLE 1. Data for patients who underwent decompression of the LFCN

\begin{tabular}{lcccc}
\hline \multicolumn{1}{c}{ Variable } & Mean & SD & Median & Range \\
\hline Age (yrs) & 50 & 8 & 54 & $27-61$ \\
\hline Duration of symptoms (yrs) & 61 & 70 & 23 & $9-223$ \\
\hline Follow-up (mos) & 27 & 18 & 25 & $5-58$ \\
\hline BMl $\left(\mathrm{kg} / \mathrm{m}^{2}\right)$ & 30.2 & 5.5 & 28.2 & $21.5-39.2$ \\
\hline
\end{tabular}

Seventeen patients ( 12 women and 5 men) underwent 19 procedures. Two men underwent LFCN decompression bilaterally.

* Interval between onset of symptoms and operation.

has not been described before. Here, we describe our technique in detail and present the results.

\section{Methods \\ Patient Population}

We performed a retrospective cohort study in a consecutive case series of patients who underwent dynamic LFCN decompression to treat iMP in the period between June 2013 and December 2017.

The indication for surgery was the presence in the LCFN area of 1) persistent, annoying pain and/or paresthesia; and 2) abnormal sensation of the skin (hypesthesia, dysesthesia, hyperesthesia, allodynia, or hyperalgesia). Patients had been previously treated elsewhere, but their symptoms were refractory to conservative measures, local injection containing steroids and anesthetic, or, occasionally, PRF.

Patients were excluded if they had prior LFCN surgery. All patients were tested for symptomatic MP based on neuropathy due to prolonged external compression, traumatic lesion in the area of the ASIS, an L2 or L3 radiculopathy, a lumbar plexopathy, a femoral neuropathy, a lower abdominal wall procedure, or an iliac bone graft harvest. All patients had ultrasound and/or MRI examinations to rule out a retroperitoneal or intrapelvic mass. Additional exclusion criteria were age $\leq 18$ years or inability to communicate in Dutch.

Seventeen patients ( 12 women) met these criteria (Table 1). In 2 patients, the LFCN was decompressed bilaterally in separate sessions after full recovery of the first operation, amounting to 19 cases.

The mean age of the patients at surgery was 50 years (SD 8, range 27-61, and median 54 years). The mean interval between onset of symptoms and operation was 61 months (SD 70, range 9-223, and median 23 months). The mean follow-up was 27 months (SD 18, range 5-58, and median 25 months). The mean BMI was $30.2 \mathrm{~kg} / \mathrm{m}^{2}$ (SD 5.5 , range $21.5-39.2$, and median $28.2 \mathrm{~kg} / \mathrm{m}^{2}$ ). The primary goal of decompression was complete relief of the pain and/or paresthesia in the LFCN area. The second aim was recovery of sensation.

Patients were sent a letter containing background information and to request permission for a phone interview about the status of the preoperatively collected variables regarding 1) pain and paresthesia and 2) skin sensation. An experienced neurologist who was not involved in the treatment used a semistructured interview by asking sim- 


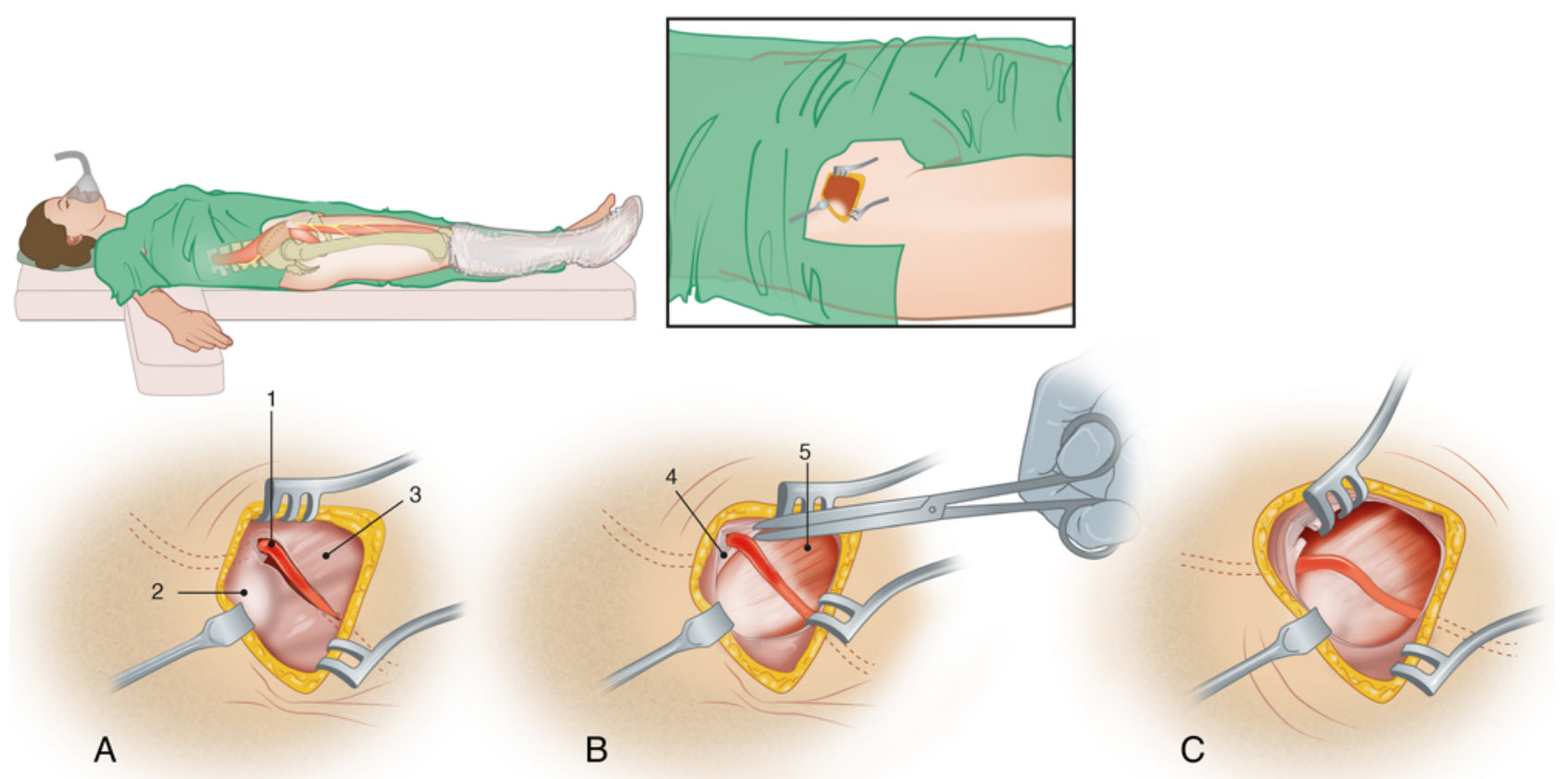

FIG. 1. Ventral decompression of the LFCN. Illustrations from a surgeon's angle of view. The leg on the affected side was positioned and draped so that it could be freely moved in full range of flexion and abduction/extension during the surgery. A: The subcutaneous fat is divided over the ASIS down to the fascia lata, and over the lateral part of the inguinal ligament and distal part of the fascia of the abdominal wall musculature. The plane ventral to the LFCN is opened by cutting the fascia lata and inferior part of the inguinal ligament in a caudocranial direction. Care is taken to leave the superior part of the inguinal ligament intact to secure the attachment of the abdominal wall musculature to the ligament. The LFCN is identified by careful blunt dissection in the fatty tissue just medial and caudal to the ASIS and lateral to the sartorius muscle. 1, LFCN; 2, ASIS; 3, fascia lata. B: If present, arcuate fibers of the inguinal ligament forming a slip medial and lateral to the LFCN and dorsally attaching to the tendinous origin of the sartorius muscle were cut. The LFCN at this level typically showed a focal indentation as well as distinct epineurial hypervascularization due to compression. 4, inguinal ligament; 5, sartorius muscle. C: The dorsal-to-ventral angulation of the LFCN over a firm fibrous band comes into view at the level medial to the ASIS and can be felt medial to the LFCN. The plane dorsal to the LFCN at the level of the ASIS consists of the fascia and musculotendinous origin of the sartorius muscle. More proximally, it consists of the fascia and origin of the sartorius muscle merging with the fascia of the iliacus muscle. More distal to the ASIS, the dorsal plane consists of the lateral aspect of the fascia of the sartorius muscle. Copyright Martijn J. A. Malessy. Published with permission.

ple and reproducible questions and using a 4-point scale (completely resolved, improved, not changed, or worsened). If not completely resolved, patients were additionally asked to score the remaining pain, paresthesia, and sensory deficit as a percentage of the preoperative level. In addition, patients were asked whether they were satisfied with the outcome of surgery (yes, no, or partially). ${ }^{1} \mathrm{~Pa}-$ tients were given the opportunity to refrain from participation by returning an answer sheet by email or telephone. All patients provided informed consent and were willing to participate.

Statistical assessment was done using ANOVA to assess the association between postoperative pain and sensation and duration of preoperative symptoms, BMI, and length of follow-up. Significance was set at $\mathrm{p} \leq 0.05$. $\mathrm{R}$ (version 3.3.3, R Foundation for Statistical Computing, R Core Team 2017) was used.

This study was performed with approval of the medical ethics committee of the LUMC.

\section{Surgical Technique for Dynamic LFCN Decompression}

All procedures were performed by a single nerve surgeon (M.J.A.M.). The surgery was performed on the ipsilateral side using loupe magnification. The operation was performed under general anesthesia on an outpatient basis. Patients were positioned supine on the operating table with the affected side close to the edge. In obese patients, the belly overlying the surgical area was shifted craniomedially and fixed with elastic adhesive tape. Sterile draping was performed such that the thigh at the affected side could be freely moved in full range of flexion and abduction/extension during the surgery (Fig. 1).

An incision of approximately $7 \mathrm{~cm}$ was made in the skin line centered over the ASIS running parallel to and over the inferior lateral extent of the inguinal ligament. The subcutaneous fat was divided down to the fascia lata, the lateral part of the inguinal ligament, and distal part of the fascia of the abdominal wall musculature. The LFCN was identified underneath the fascia lata caudal to the attachment of the inguinal ligament to the ASIS (Fig. 1A). The attachment of the fascia lata to the inguinal ligament was opened from distal to proximal. The inferior part of the inguinal ligament was cut. Occasionally, arcuate fibers of the inguinal ligament were present, forming a slip medial and lateral to the LFCN. If present, the slip was cut medial to the LFCN (Fig. 1B). At this stage of the surgery, a dorsal-to-ventral angulation of the LFCN was seen as it emerges from the pelvis. Here, it runs over a firm fibrous 

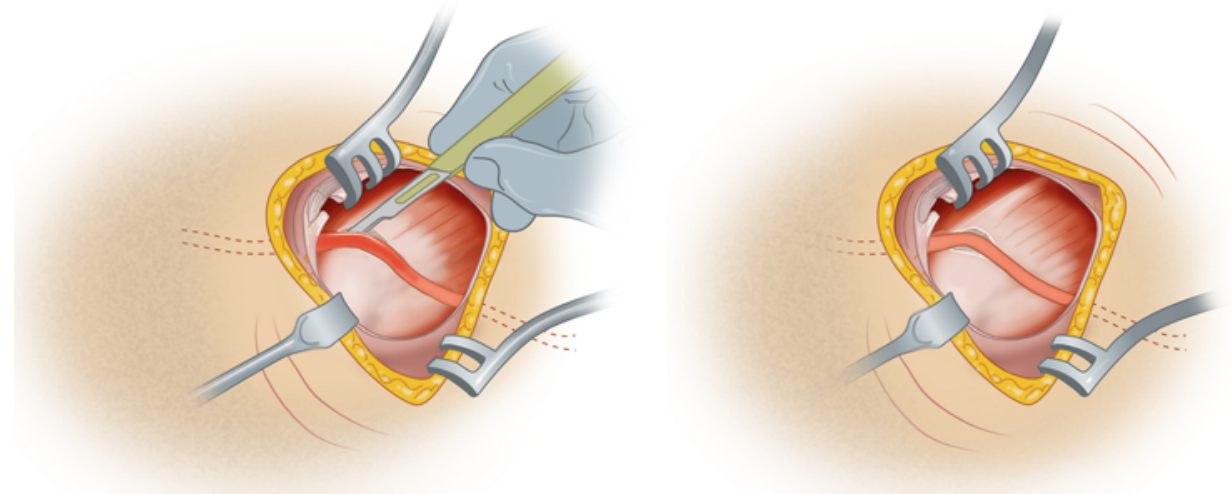

FIG. 2. Dorsal decompression of the LFCN. Left: Dorsal decompression is started with the leg in neutral by cutting the fascia of the sartorius muscle at the level of the angulation. Right: The LFCN shifts somewhat posterior and its angulation becomes less pronounced. In neutral, it appears that the dorsal plane of the LFCN is relaxed. Seemingly, dorsal decompression has been adequately executed. Copyright Martijn J. A. Malessy. Published with permission.

band at the level medial to the ASIS. This band is formed by the fascia and musculotendinous origin of the sartorius muscle (Fig. 1C). Dorsal decompression was performed by cutting the fascia of the sartorius muscle at the level of the angulation (Fig. 2).

Subsequently, dynamic testing was performed. To assess whether complete ventral decompression was obtained, the thigh was brought in complete flexion and abduction, and the space ventral to the LFCN was palpated with the top of the index finger (Fig. 3 left). If fibers compressed the LFCN, they were additionally cut until it was adequately freed.

Thereafter, the thigh was brought in full range of extension and abduction to assess whether dorsal compression of the LFCN still occurred. The leg was thereby positioned next to and below the level of the operating table (Fig. 3 right). During extension, we observed the movement of the LFCN and assessed the tissue pressure dorsal to the LFCN
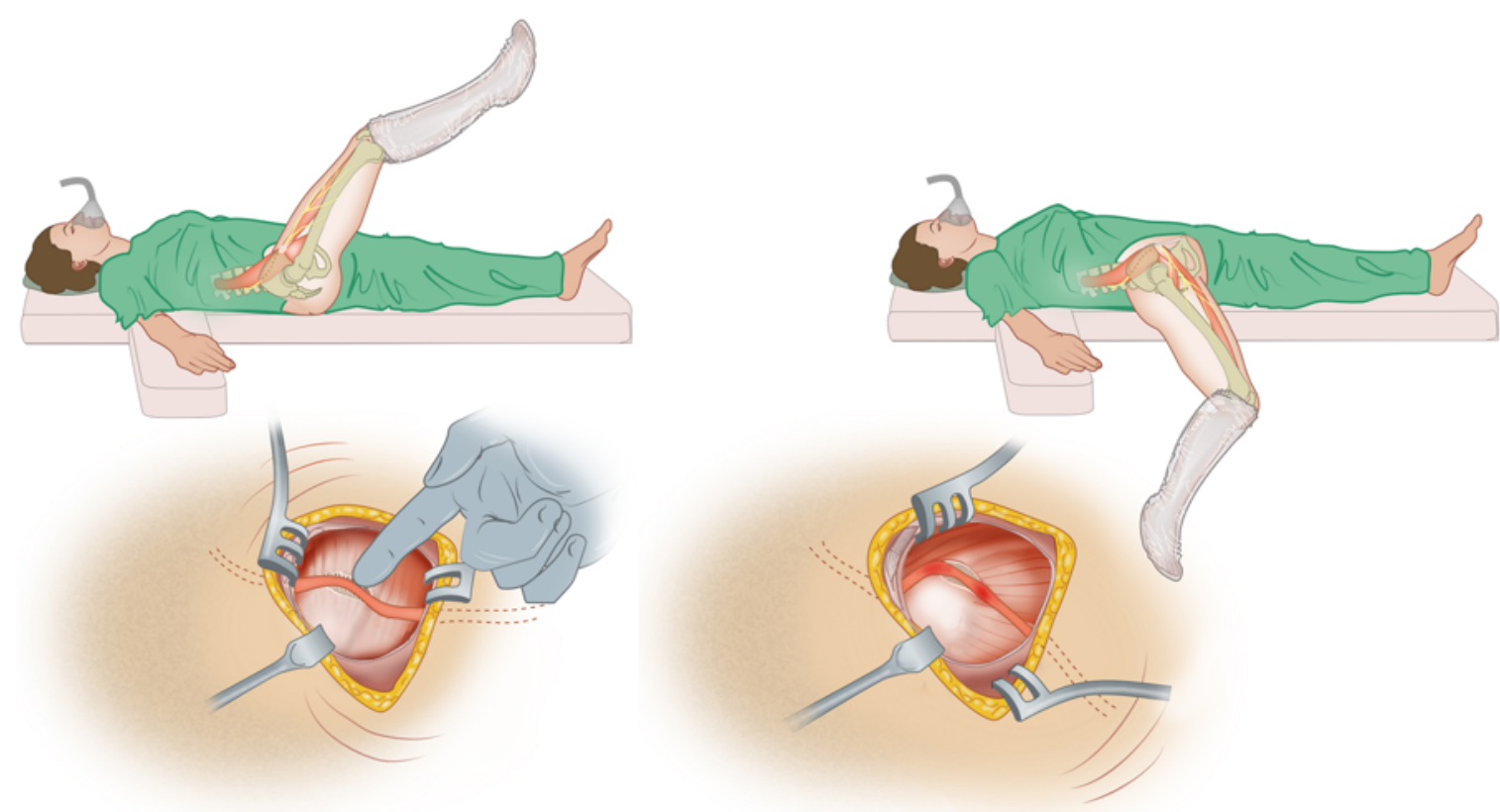

FIG. 3. Dynamic testing of decompression of the LFCN. Left: Dynamic testing to assess whether the ventral decompression was sufficiently performed by palpating close to the LFCN with the top of the index finger. When the thigh was flexed, ample space was usually present between the LFCN and the remaining inguinal ligament, and the distal fascia lata did not cause any compression. If ventral fibers still compressed the LFCN, they were additionally cut until the LFCN was adequately freed. Right: The thigh was brought in extension/abduction next to and below the level of the operating table. The LFCN was gradually pushed anteriorly during the process of gentle extension and the angulation became more pronounced. The pressure exerted by the plane dorsal to the LFCN increased and caused compression at varying locations caused by tightening of fibers. These could be fibers of the musculotendinous origin of the sartorius at the level of the ASIS, iliac fascia proximal to the ASIS, or the lateral aspect of the fascia of the sartorius muscle distal to the ASIS. Copyright Martijn J. A. Malessy. Published with permission. 

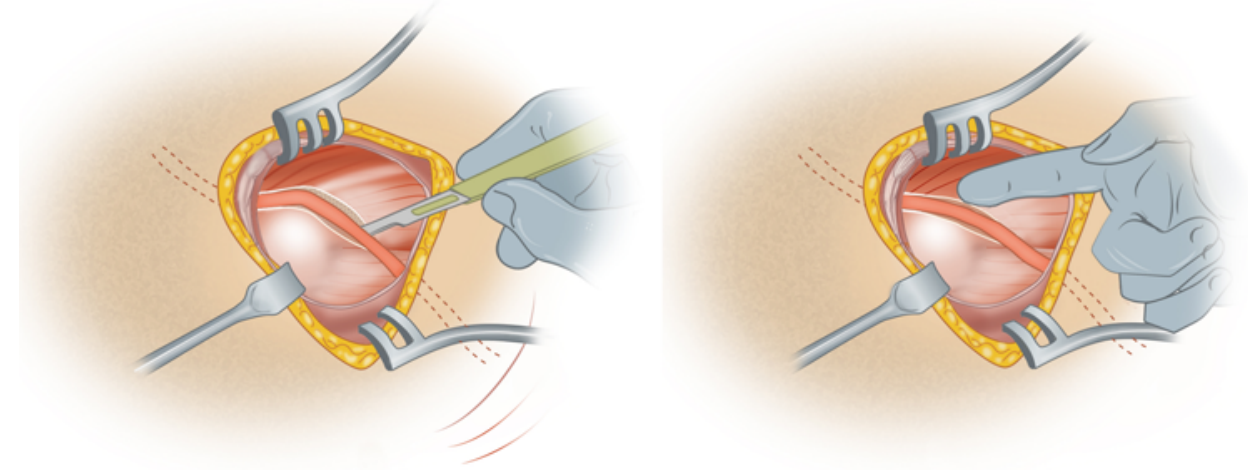

FIG. 4. Dynamic decompression of the LFCN. Left: Fibers dorsal to the LFCN that became tight during extension and caused compression of the LFCN were additionally cut, thereby obtaining complete dorsal decompression. We call this dynamic decompression. Right: Complete dorsal decompression and release with posterior shifting of the LFCN in full range of extension and abduction, a neutralized angulation, and soft tissue dorsal to the LFCN. During dynamic testing, shifting of the LFCN in the frontal plane from lateral to medial or vice versa over the ASIS was not observed. Copyright Martijn J. A. Malessy. Published with permission.

with the top of the index finger due to tightening of the fibers dorsal to the LFCN.

Compression was present if 1) the LFCN shifted anteriorly; 2) the angulation became more pronounced; and 3) a high pressure of the tissue in the plane dorsal to the LFCN was palpable (Fig. 3 right). The compression-causing fibers were then cut (Fig. 4 left). Complete dorsal decompression was considered to have been obtained if, in full range of extension and abduction, a posterior shift of the LFCN was observed, the angulation was neutralized, and the tissue dorsal to the LFCN felt soft (Fig. 4 right). The LFCN was then released and left in situ without any additional fixation. Internal neurolysis was not performed. A (vacuum) drain was not used. After surgery, same-day mobilization of the patient was encouraged to ensure sliding of the nerve.

\section{Results}

All patients underwent further decompression by cutting compression-causing fibers dorsal to the LFCN that were identified with dynamic movement. In 17 of the 19 cases (89\%), the pain and/or paresthesia completely resolved. The patients in the remaining 2 cases (11\%) experienced pain reduction of $70 \%$ and $80 \%$ (Table 2). Sensation completely recovered in 13 of the 19 cases $(69 \%)$ and

TABLE 2. Results of dynamic LCFN decompression

\begin{tabular}{|c|c|c|c|}
\hline & $\begin{array}{c}\text { Completely } \\
\text { Resolved (\%) }\end{array}$ & $\begin{array}{l}\text { Improved } \\
(\%)\end{array}$ & $\begin{array}{c}\text { Not Changed } \\
(\%)\end{array}$ \\
\hline Pain \&/or paresthesia & $17(89)$ & $2(11)^{*}$ & \\
\hline Sensation in LFCN area & $13(69)$ & $5(26) \dagger$ & $1(5) \ddagger$ \\
\hline \multicolumn{4}{|c|}{$\begin{array}{l}\text { No patient experienced worsening. } \\
\text { * Patients in these } 2 \text { cases estimated their improvement as } 70 \% \text { and } 80 \% \text { less } \\
\text { pain. } \\
\dagger \text { In these } 5 \text { cases, an area of hypesthesia remained. None of the patients ex- } \\
\text { perienced any dysesthesia, hyperesthesia, allodynia, or hyperalgesia. Patients } \\
\text { in } 4 \text { of the } 5 \text { cases indicated a sensory improvement of more than } 75 \% \text {, and } 1 \\
\text { had } 50 \% \text { improvement. } \\
\ddagger \text { Persistent hypesthesia and mild hyperesthesia. }\end{array}$} \\
\hline
\end{tabular}

improved in 5 of the 19 cases (26\%). In these 5 patients, an area of hypesthesia remained, but none of the patients experienced any dysesthesia, hyperesthesia, allodynia, or hyperalgesia. Four of the 5 cases indicated a sensory improvement of more than $75 \%$, and 1 had $50 \%$ improvement. Sensation remained unchanged in 1 case $(5 \%)$ with persisting hypesthesia and mild hyperesthesia. All of the patients showed improvement in carrying out daily activities, and all were satisfied with the surgery.

There was no significant impact of duration of preoperative symptoms, BMI, and length of follow-up on postoperative outcome. All p values were well above 0.05 .

We consistently found either type I or II anatomy of the $\mathrm{LFCN}^{43}$ during surgery. LFCN shifting in the frontal plane from lateral to medial or over the ASIS was not observed during dynamic testing. Surgical time did not exceed 1.5 hours. Postoperatively, there was 1 complication, temporary seroma development that did not require further intervention.

\section{Discussion}

Idiopathic MP may cause annoying pain, paresthesia, and abnormal sensation in the area of the LFCN. These complaints may affect daily activities and sleep to such an extent that treatment is indicated. Usually, conservative measures and local infiltration are tried first. Surgery is considered if an adequate response is not obtained.

We performed dynamic decompression of the LFCN, after which the vast majority of patients became completely pain free, with the remaining patients experiencing considerable pain reduction (Table 2).

In addition, sensation in the LFCN skin area completely recovered in more than two-thirds of the cases, and good improvement occurred in the remaining cases. Preexisting annoying dysesthesia, hyperesthesia, allodynia, or hyperalgesia, if any, disappeared in all patients. All patients were satisfied and frequently spontaneously expressed their enthusiasm about the outcome of the surgery. The outcomes of our patients are better than those published to date in which pain relief was obtained in $30 \%$ to $82 \%$ of the patients. ${ }^{4,29,38,39,44}$ 


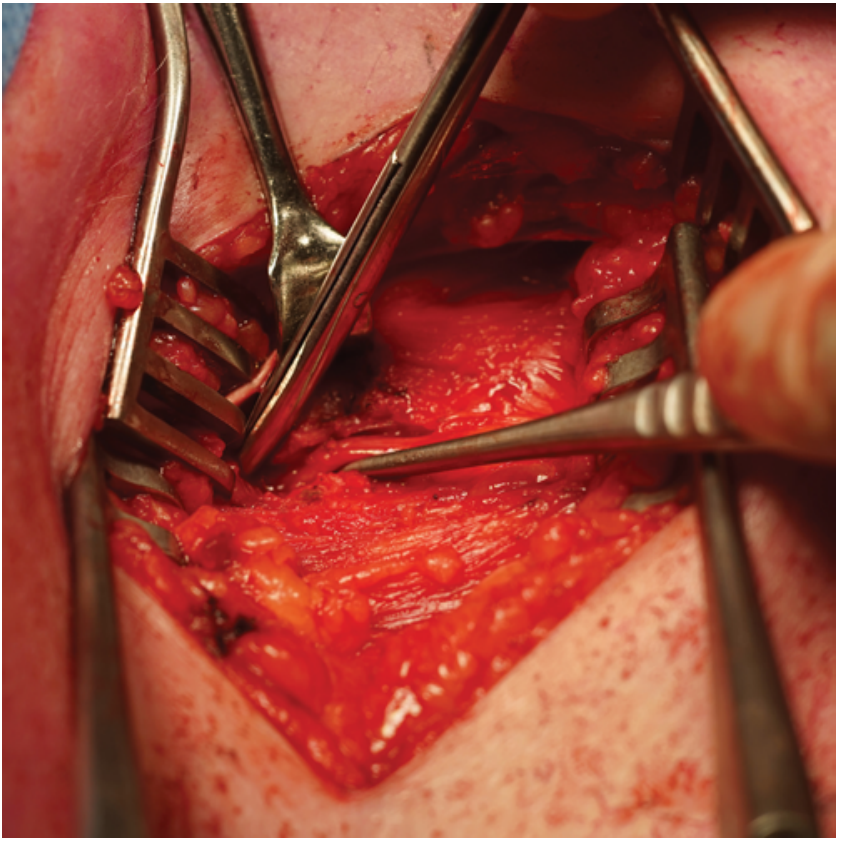

FIG. 5. Intraoperative photograph of the marked dorsal-to-ventral angulation of the LCFN as it emerges from the pelvis. The leg is in neutral position. The LFCN is indicated by tweezers, which are pushed against the medial aspect of the ASIS. See Fig. 1C for further orientation.

Comparing our results with those of others turns out to be difficult because few studies are suitable for comparison. ${ }^{4,38,39}$ Our technique differs from those described so far in that we address the LFCN in a dynamic fashion. During surgery, we assess whether the LFCN is sufficiently decompressed in the full range of motion of the thigh (Figs. 1-6). In one study, the plane ventral to the LFCN was sectioned, and good results were achieved in $64 \%$ of the patients. ${ }^{4}$ In the other two studies, the plane dorsal to the LFCN was additionally cut. Complete pain relief was thereby obtained in $73 \%$ and $82 \%$ of patients. ${ }^{38,39}$

By moving the leg in extension and abduction, we observed that fibers located dorsal to the LFCN tighten and cause compression. It might seem that decompression performed with the leg in a neutral position was adequately performed, but then the compressing effect of tightening of fibers is missed. Cutting these fibers (Fig. 6) likely is the distinctive factor that positively distinguishes our results from those of others. It is simple to achieve and should, therefore, not be left undone. Moreover, it did not lead to complaints in the surgical area. To exactly determine to what extent dynamic decompression has contributed to the good results, a study must be performed in which 2 surgical procedures are prospectively randomized with blinding of both the patient and the outcome assessor. One surgical procedure should then be the classic static fashion ${ }^{38,39}$ and the other the dynamic decompression.

Deducing the etiology of iMP from the extent to which decompression was performed and subsequently the results, one might conclude that not only the ventral plane ${ }^{4}$ plays a role in the compression of the LFCN, but the dorsal plane as well. ${ }^{38,39}$ Our study further adds to the understand-

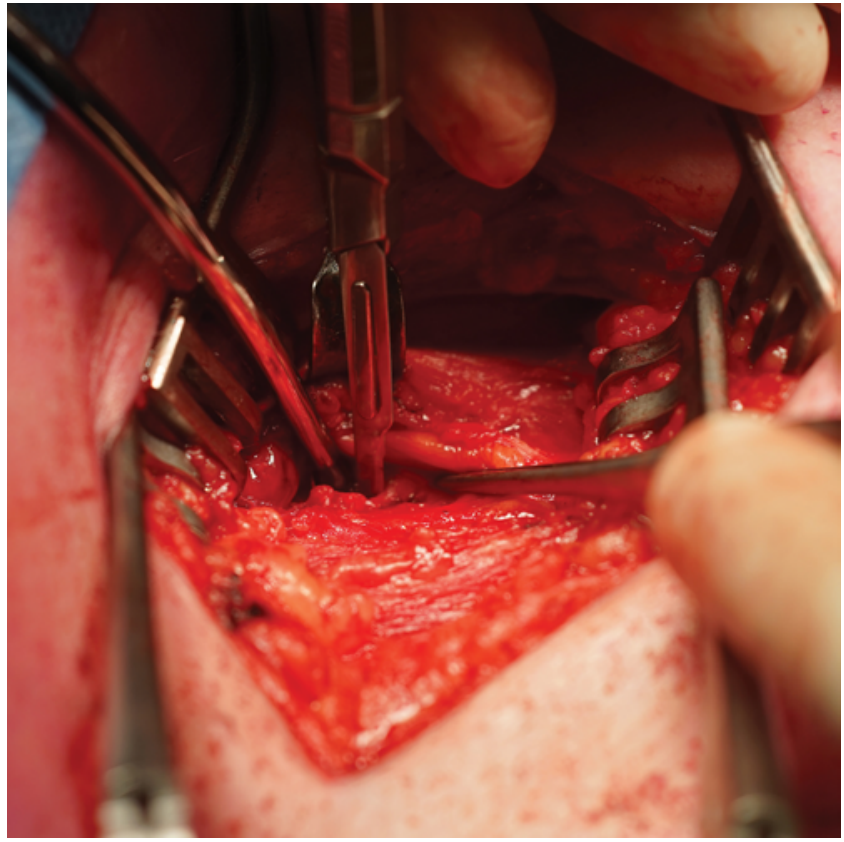

FIG. 6. Intraoperative photograph of additional decompression of the LFCN. The fascia of the sartorius muscle dorsal to the LFCN at the level of the angulation has already been cut. Musculotendinous fibers of the origin of the sartorius muscle at the level the ASIS were identified to cause compression while tightening during extension. These fibers were additionally cut, thereby obtaining complete dorsal decompression. See Fig. 4 left for further orientation

ing of the etiology in that the dorsal plane plays a role in the dynamics of the compression. Postural variations in MP have been attributed to "the pincer" formed by 2 bands of Poupart's ligament. ${ }^{26}$ It was Stookey in 1928, however, who noted the marked angulation of the LCFN as it emerged from the pelvis medial to the anterior spine (Fig. 5) and its significance in the dynamic pathophysiology of iMP. ${ }^{40}$ He wrote, "The nerve at this point forms an abrupt angle which is considerably increased by extension and lessened by flexion of the thigh. The cause of iMP is the abnormal sharp angulation of the LFCN as it leaves the pelvis with continuous trauma to the nerve in movements of the thigh, especially in standing and walking." These observations, however, did not lead him to decompress the nerve and neutralize the angulation. Instead, he sectioned the nerve at the exit from the pelvis.

Thus, our technique is a translation of Stookey's observation into actual surgical handling. We support his opinion regarding the dynamic contribution of the plane dorsal to the LFCN in the etiology of idiopathic MP because of 1) our intraoperative observation of increasing dorsal compression of the LFCN during retroflexion by stiffening of a dorsal fibrous band and 2) the good results obtained if dorsal dynamic decompression in extension is completed. Actually, the LFCN thereby shifts from ventral to dorsal. Surgical transposition for decompression of the LFCN in the frontal plane has been proposed. ${ }^{14,17,21,22,28}$ First, the LFCN was transposed from medial to lateral. ${ }^{28}$ Later, it was found more logical to transpose the nerve from lateral to medial. ${ }^{14,17,21,22}$ Intraoperatively, we did not observe shift- 
ing of the LFCN in the frontal plane during movement after dynamic decompression. Notably, riding over the ASIS did not occur, and therefore there is no rationale for medial transposition. Medial fixation with a stitch ${ }^{19}$ might even be contraindicated.

Surgical technique is not the only factor that affects outcome. Duration of symptoms, obesity, and anatomical variations also affect outcome, making it difficult to compare outcomes. In our series, the mean interval between onset and surgery was approximately 5 years. We did not find an association between duration of symptoms, postoperative pain relief, and recovery of sensation. It is remarkable that even after an interval of many years pain completely disappeared and sensation returned to normal. Others found significantly better results if decompression was performed within 6 months of the first complaints ${ }^{4}$ Histopathological examination of LFCN biopsies under the area of compression showed reduced myelinated nerve fiber density. ${ }^{5}$ Such changes are likely associated with positive sensory phenomena such as pain and paresthesia found in entrapment neuropathy.$^{36}$ Obviously, we could not study potential histopathological nerve changes in our patients. However, we observed focal indentation of the LFCN befitting compression, as well as distinct epineurial hypervascularization. It seems reasonable to postulate that pathologic changes were present in the LFCN of some of our patients. In view of the beneficial effect of surgery, these must have been largely reversible. In view of the prompt postoperative pain reduction, myelination was likely not the underlying recovery process, which would have taken longer to complete.

Obesity was found to be significantly associated with a worse long-term outcome. ${ }^{38}$ We and others ${ }^{4}$ did not find this association. Obesity has also been suggested as a factor indirectly related to LFCN compression. It was postulated that the weight of the abdominal fat causes lowering of the inguinal ligament. ${ }^{12}$ In view of the dynamics of compression, this might not be the only causality. We postulate that obese people might develop a relatively bulky iliac muscle to bear body weight that in turn contributes to the development of dorsal LFCN compression. This mechanism might also play a role in slim individuals who subject themselves to a rigorous training schedule. In broader terms, iMP may be the result of imperfect evolution. Initially, while walking on all fours, the angulation of the LFCN did not compromise the nerve. By standing up and walking bipedally, the LFCN course shifted to the outside of a pivot, thereby becoming susceptible for compression. Analog to these changes, there is a resemblance with the compression of the ulnar nerve at the level of the cubital tunnel.

Anatomical variations of the $\mathrm{LFCN}^{43}$ may account for failure of decompression and differences in reported outcome. Seven different types of exit of the LFCN from the pelvis were described. Quantitatively, only types I and II are relevant, comprising more than $90 \%$ of the anatomy. In both of these types, the LFCN exits the pelvis medial to the ASIS and under or through the inguinal ligament where the decompression is performed. ${ }^{43}$ Anatomical variations have been found by others during surgery, ${ }^{4}$ but the more common type I and II courses of the LFCN, as we consistently found, were typically prone to compression. ${ }^{1}$ We do not feel the urge to use the suprainguinal retroperitoneal approach, which was developed for more easy identification of the LFCN. ${ }^{1}$ In view of the recent developments in the area of ultrasound, the advantage of this approach seems limited. In addition, it might be more difficult to obtain adequate dorsal decompression via this approach.

For quite some time there has been an ongoing discussion whether the LFCN should be decompressed or cut to relieve symptoms of iMP.11,18 This study shows that good results can be obtained with decompression in the vast majority of patients, provided that a proper surgical technique is mastered. In a direct comparison of nonrandomized patients, only $37 \%$ of patients had pain relief following decompression. ${ }^{10}$ Subsequently, these substandard results were used to propagate neurectomy. Only a randomized controlled trial (RCT) can address which technique should become standard: decompression or neurectomy ${ }^{35}$ Results of such an RCT can only be taken seriously if $80 \%-90 \%$ of patients are pain free in the decompression group. We consider sensation as an important outcome measure as well. Following neurectomy sensation is completely lost, but after decompression it can substantially recover. In view of our results, it may be clear that we do not see the need for an RCT. There is no rationale for cutting a nerve in a compression syndrome if adequate surgical decompression provides good symptom relief. Last but not least, the consequences of neurectomy can be devastating, however infrequently it may occur. Birch et al. unavailingly sought to relieve intractable pain in 4 patients after sectioning of the LFCN elsewhere. ${ }^{6}$ Additionally, annoying numbness may develop, which cannot be neglected as a negative side effect of neurectomy. ${ }^{24,25,37,42}$

The current treatment algorithm for iMP recommends a step-by-step approach if conservative measures fail. The steps consist of local infiltration with steroids and anesthetics, followed by PRF and then spinal cord stimulation. ${ }^{34}$ The scientific base of the algorithm is small. Data on the use of PRF for iMP are lacking, and thus the efficacy is unknown. ${ }^{9}$ Reports on spinal cord stimulation for iMP are rare. ${ }^{3}$ The role assigned to surgery in the algorithm is negligible. Based on our results and those of others, ${ }^{38,39}$ the role of surgical decompression in treating patients with iMP needs to be revised. We feel that patients who do not respond to conservative measures and local infiltration should be promptly referred for decompression, preferably within 6 months of onset. Decompression, if mastered well, is not a burdensome surgical procedure.

The strengths of this study are as follows. 1) A relatively large consecutive case series of patients exclusively with iMP were treated by a single surgeon. 2) New insights of surgical decompression were described and illustrated in detail, allowing proper interpretation of the results and future comparison. 3) Three outcome parameters were assessed by an independent neurologist with a long follow-up. A weakness of our study is the exactness of outcome measurement. The patients' pain levels were not systematically scored before and after surgery, for instance, on a visual analog or numeric rating scale. Following decompression, however, the vast majority of patients became completely pain free, which was in clear contrast to the preoperative burden. Additionally, quantitative examination of sensation in the LFCN area before and after surgery was not 
performed, e.g., with Semmes-Weinstein monofilaments. Although sensation recovery was self-reported, it is likely to be accurate and occurred in more than two-thirds of the cases.

\section{Conclusions}

LFCN decompression intuitively seems to be a straightforward procedure, but it has not been described in great detail. Like in many surgical procedures, the devil is in the detail. In all other techniques described so far, the LFCN is decompressed in a fixed supine position with the thigh neutral. We observed that bands in the dorsal plane tighten during extension, which causes compression of the LFCN at various locations. This tightening is missed if not tested dynamically. Dynamic decompression of the LFCN is an effective technique to treat iMP. Most patients become completely pain free and sensation recovers considerably.

\section{References}

1. Alberti O, Wickboldt J, Becker R: Suprainguinal retroperitoneal approach for the successful surgical treatment of meralgia paresthetica. J Neurosurg 110:768-774, 2009

2. Antoniadis G, Braun V, Rath S, Moese G, Richter HP: [Meralgia paraesthetica and its surgical treatment.] Nervenarzt 66:614-617, 1995 (Ger)

3. Barna SA, Hu MM, Buxo C, Trella J, Cosgrove GR: Spinal cord stimulation for treatment of meralgia paresthetica. Pain Physician 8:315-318, 2005

4. Benezis I, Boutaud B, Leclerc J, Fabre T, Durandeau A: Lateral femoral cutaneous neuropathy and its surgical treatment: a report of 167 cases. Muscle Nerve 36:659-663, 2007

5. Berini SE, Spinner RJ, Jentoft ME, Engelstad JK, Staff NP, Suanprasert N, et al: Chronic meralgia paresthetica and neurectomy: a clinical pathologic study. Neurology 82:15511555,2014

6. Birch R, Bonney G, Wynn Parry CB: Surgical Disorders of the Peripheral Nerves. Edinburgh: Churchill Livingstone, 1998

7. Boyd KU, Brown JM: Injury and compression neuropathy in the lower extremity, in Mackinnon SE (ed): Nerve Surgery. New York: Thieme, 2015, pp 338-390

8. Bruyere A, Hidalgo Diaz JJ, Vernet P, Salazar Botero S, Facca S, Liverneaux PA: Technical feasibility of robot-assisted minimally-invasive neurolysis of the lateral cutaneous nerve of thigh: about a case. Ann Chir Plast Esthet 61:872-876, 2016

9. Chang MC: Efficacy of pulsed radiofrequency stimulation in patients with peripheral neuropathic pain: a narrative review. Pain Physician 21:E225-E234, 2018

10. de Ruiter GCW, Kloet A: Comparison of effectiveness of different surgical treatments for meralgia paresthetica: results of a prospective observational study and protocol for a randomized controlled trial. Clin Neurol Neurosurg 134:7-11, 2015

11. de Ruiter GCW, Kloet F: Anatomical considerations on transposition of the lateral femoral cutaneous nerve. Clin Anat [epub ahead of print], 2018 (Letter)

12. Deal CL, Canoso JJ: Meralgia paresthetica and large abdomens. Ann Intern Med 96:787-788, 1982

13. Ducic I, Dellon AL, Taylor NS: Decompression of the lateral femoral cutaneous nerve in the treatment of meralgia paresthetica. J Reconstr Microsurg 22:113-118, 2006

14. Ducic I, Iorio ML: Modified approach for lateral femoral cutaneous nerve decompression in patients with meralgia paresthetica. Plast Reconstr Surg 127:82e-83e, 2011

15. Edelson R, Stevens P: Meralgia paresthetica in children. J Bone Joint Surg Am 76:993-999, 1994
16. Filler AG, Gilmer-Hill H: Piriformis syndrome, obturator internus syndrome, pudendal nerve entrapment, and other pelvic entrapments, in Winn HR (ed): Youmans Neurological Surgery, ed 6. Philadelphia: Elsevier Saunders, 2011, Vol 3, pp 2447-2455

17. Hanna A: Transposition of the lateral femoral cutaneous nerve. J Neurosurg [epub ahead of print April 13, 2018; DOI: 10.3171/2017.8.JNS171120]

18. Hanna A, Hanna B: Response to: Anatomical considerations on transposition of the lateral femoral cutaneous nerve. Clin Anat [epub ahead of print], 2018 (Letter)

19. Hanna AS: Lateral femoral cutaneous nerve transposition: Renaissance of an old concept in the light of new anatomy. Clin Anat 30:409-412, 2017

20. Heinen C, Kretschmer T: Compressive lesions of the lower limb and trunk, in Socolovsky M, Rasulic L, Midha R, et al (eds): Manual of Peripheral Nerve Surgery. Stuttgart: Thieme, 2018, pp 115-127

21. Keegan JJ, Holyoke EA: Meralgia paresthetica. An anatomical and surgical study. J Neurosurg 19:341-345, 1962

22. Kempe LG: Surgery of peripheral nerves, in Kempe LG (ed): Operative Neurosurgery. Berlin: Springer, 1970, Vol 2, pp 208-210

23. Khalil N, Nicotra A, Rakowicz W: Treatment for meralgia paraesthetica. Cochrane Database Syst Rev 12:CD004159, 2012

24. Kline DG, Hudson AR: Nerve Injuries. Operative Results for Major Nerve Injuries, Entrapments, and Tumors. Philadelphia: W.B. Saunders Company, 1995

25. Kopell HP: Lower extremity lesions, in Omer GE, Spinner M (eds): Management of Peripheral Nerve Problems. Philadelphia: W.B. Saunders Company, 1980, pp 626-638

26. Learmonth JR: The principle of decompression in the treatment of certain diseases of peripheral nerves. Surg Clin North Am 13:905-913, 1933

27. Lee FC: Meralgia paresthetica. Int Clin 1:210-230, 1936

28. Lee FC: An osteoplastic neurolysis operation for the cure of meralgia paraesthetica. Ann Surg 113:85-94, 1941

29. Macnicol MF, Thompson WJ: Idiopathic meralgia paresthetica. Clin Orthop Relat Res (254):270-274, 1990

30. Naff NJ, Belzberg AJ: Unusual peripheral nerve entrapments, in Kaye AH, Black PM (eds): Operative Neurosurgery. London: Churchill Livingstone, 2000, Vol 2, pp 2147-2153

31. Nahabedian MY, Dellon AL: Meralgia paresthetica: etiology, diagnosis, and outcome of surgical decompression. Ann Plast Surg 35:590-594, 1995

32. Neisser E, Pollack C: Beitrag zur Kenntnis der RothBernhardt'schen Meralgie und ihrer operativen Behandlung. Mitteilungen aus den Grenzgebieten der Medizin und Chirurgie 10:453-461, 1902

33. Nouraei SA, Anand B, Spink G, O'Neill KS: A novel approach to the diagnosis and management of meralgia paresthetica. Neurosurgery 60:696-700, 2007

34. Patijn J, Mekhail N, Hayek S, Lataster A, van Kleef M, Van Zundert J: Meralgia paresthetica. Pain Pract 11:302-308, 2011

35. Payne R, Seaman S, Sieg E, Langan S, Harbaugh K, Rizk E: Evaluating the evidence: is neurolysis or neurectomy a better treatment for meralgia paresthetica? Acta Neurochir (Wien) 159:931-936, 2017

36. Schmid AB, Bland JD, Bhat MA, Bennett DL: The relationship of nerve fibre pathology to sensory function in entrapment neuropathy. Brain 137:3186-3199, 2014

37. Sinisi M: Management of entrapment neuropathies, in Quinones-Hinojosa A (ed): Schmidek and Sweet. Operative Neurosurgical Technique. Indications, Methods and Results, ed 6. Philadelphia: Elsevier Saunders, 2012, Vol 2, pp 2299-2207

38. Siu TL, Chandran KN: Neurolysis for meralgia paresthetica: an operative series of 45 cases. Surg Neurol 63:19-23, 2005

39. Son BC, Kim DR, Kim IS, Hong JT, Sung JH, Lee SW: Neu- 
rolysis for meralgia paresthetica. J Korean Neurosurg Soc 51:363-366, 2012

40. Stookey B: Meralgia paraesthetica: etiology and surgical treatment. JAMA 90:1705-1707, 1928

41. Teng P: Meralgia paresthetica. Bull Los Angeles Neurol Soc 37:75-83, 1972

42. Tindall SC: Surgical management of thoracic outlet syndrome and peripheral entrapment neuropathies, in Schmidek HH, Sweet WH (eds): Schmidek \& Sweet Operative Neurosurgical Techniques: Indications, Methods, and Results, ed 4. Philadelphia: Saunders, 2000, Vol 2, pp 2173-2182

43. Tomaszewski KA, Popieluszko P, Henry BM, Roy J, Sanna B, Kijek MR, et al: The surgical anatomy of the lateral femoral cutaneous nerve in the inguinal region: a meta-analysis. Hernia 20:649-657, 2016

44. van Eerten PV, Polder TW, Broere CA: Operative treatment of meralgia paresthetica: transection versus neurolysis. Neurosurgery $37: 63-65,1995$

\section{Disclosures}

The authors report no conflict of interest concerning the materi- als or methods used in this study or the findings specified in this paper.

\section{Author Contributions}

Conception and design: all authors. Acquisition of data: Malessy, Eekhof. Analysis and interpretation of data: all authors. Drafting the article: Malessy. Critically revising the article: all authors.

Reviewed submitted version of manuscript: all authors. Approved the final version of the manuscript on behalf of all authors:

Malessy. Statistical analysis: Malessy. Administrative/technical/ material support: Malessy. Study supervision: Malessy.

\section{Correspondence}

Martijn J. A. Malessy: Leiden Nerve Center, Leiden, The Netherlands.malessy@lumc.nl. 